\title{
Lysine demethylase 3a in craniofacial and neural development during Xenopus embryogenesis
}

\author{
HYUN-KYUNG LEE ${ }^{1 *}$, TAYABA ISMAIL $^{1 *}$, CHOWON KIM $^{1}$, YOUNI KIM ${ }^{1}$, \\ JEEN-WOO PARK ${ }^{1}$, OH-SHIN KWON ${ }^{1}$, BEOM-SIK KANG ${ }^{1}$, DONG-SEOK LEE ${ }^{1}$, \\ TAEJOON KWON ${ }^{2}$, TAE JOO PARK ${ }^{2}$ and HYUN-SHIK LEE ${ }^{1}$
}

\begin{abstract}
${ }^{1}$ Cell and Matrix Research Institute, Kyungpook National University-Center for Nonlinear Dynamics, School of Life Sciences, Brain Korea 21 Plus Kyungpook National University Creative BioResearch Group, College of Natural Sciences, Kyungpook National University, Daegu 41566; ${ }^{2}$ School of Life Sciences, Ulsan National Institute of Science and Technology, Ulsan 44919, Republic of Korea
\end{abstract}

Received August 6, 2018; Accepted December 5, 2018

DOI: $10.3892 / \mathrm{ijmm} .2018 .4024$

\begin{abstract}
Epigenetic modifier lysine demethylase 3a (Kdm3a) specifically demethylates mono- and di-methylated ninth lysine of histone 3 and belongs to the Jumonji domain-containing group of demethylases. Kdm3a serves roles during various biological and pathophysiological processes, including spermatogenesis and metabolism, determination of sex, androgen receptor-mediated transcription and embryonic carcinoma cell differentiation. In the present study, physiological functions of Kdm3a were evaluated during embryogenesis of Xenopus laevis. Spatiotemporal expression pattern indicated that $k d m 3 a$ exhibited its expression from early embryonic stages until tadpole stage, however considerable increase of $k d m 3 a$ expression was observed during the neurula stage of Xenopus development. Depleting $k d m 3 a$ using $k d m 3 a$ antisense morpholino oligonucleotides induced anomalies, including head deformities, small-sized eyes and abnormal pigmentation. Whole-mount in situ hybridization results demonstrated that $k d m 3 a$ knockdown was associated with defects in neural crest migration. Further, quantitative polymerase chain
\end{abstract}

Correspondence to: Professor Hyun-Shik Lee, Cell and Matrix Research Institute, Kyungpook National University-Center for Nonlinear Dynamics, School of Life Sciences, Brain Korea 21 Plus Kyungpook National University Creative BioResearch Group, College of Natural Sciences, Kyungpook National University, 80 Daehak-ro, Daegu 41566, Republic of Korea

E-mail: leeh@knu.ac.kr

Professor Tae Joo Park, School of Life Sciences, Ulsan National Institute of Science and Technology, 50 UNIST-gil, Ulsan 44919, Republic of Korea

E-mail: parktj@unist.ac.kr

${ }^{*}$ Contributed equally

Key words: lysine demethylase 3a, histone demethylase, Xenopus laevis, craniofacial development, neural crest reaction revealed abnormal expression of neural markers in $k d m 3 a$ morphants. RNA sequencing of $k d m 3 a$ morphants indicated that $k d m 3 a$ was implicated in mesoderm formation, cell adhesion and metabolic processes of embryonic development. In conclusion, the results of the present study indicated that $\mathrm{Kdm} 3 \mathrm{a}$ may serve a role in neural development during Xenopus embryogenesis and may be targeted for treatment of developmental disorders. Further investigation is required to elucidate the molecular mechanism underlying the regulation of neural development by Kdm3a.

\section{Introduction}

The complex process of embryogenesis involves tight regulation of gene expression, which is controlled by different genetic and epigenetic modifications $(1,2)$. Post-translational modifications of histones, including acetylation, methylation, ubiquitination and phosphorylation, are important epigenetic modifications regulating gene expression (3).

Methylation of lysine residues of histone molecules is an important part of epigenetics and is involved in the control of gene expression (4). The methylation state of a histone around a gene determines whether it is transcriptionally active or not; histone methylation is strictly controlled by histone lysine methyltransferases and demethylases (KDMs) that are responsible for transfer and removal of a methyl group (5). Methylation of histone H3 lysine 9 (H3K9) is a distinctive feature of transcriptionally repressed genes in eukaryotic chromatin $(4,6)$. H3K9, which can be mono-, di-and tri-methylated (me1, me2 and me3, respectively), is well characterized for its roles in embryonic development (7). H3K9me3 is present in heterochromatin compartments (4) while $\mathrm{H} 3 \mathrm{~K} 9 \mathrm{me} 2$ is present in euchromatin regions of eukaryotic chromatin (1). The methylation status of $\mathrm{H} 3 \mathrm{~K} 9$ is maintained by two groups of KDMs containing Jumonji-C (JmjC) domains (8). Demethylases of the Kdm4 family catalyze $\mathrm{H} 3 \mathrm{~K} 9$ me $2 / 3$ demethylation, while demethylation of $\mathrm{H} 3 \mathrm{~K} 9 \mathrm{mel} / 2$ is catalyzed by $\mathrm{Kdm} 3 \mathrm{a}$ (9-11).

Lysine demethylase $3 a(\mathrm{Kdm} 3 \mathrm{a})$, also known as jumonji domain containing 1A, JMJD1A, KIAA0742 and testis-specific gene $\mathrm{A}$, is a JmjC-domain containing demeth- 
ylase (8). In addition to the JmjC domain, $\mathrm{Kdm} 3$ a contains a zinc finger domain and LXXLL motif; these domains/motifs are specific for steroid hormone receptor interactions $(11,12)$. $\mathrm{Kdm} 3 \mathrm{a}$ catalyzes demethylation of $\mathrm{H} 3 \mathrm{~K} 9 \mathrm{mel} / 2$ and serves a role in the activation of gene transcription (8). The catalytic activity of Kdm3a, similar to other JmjC domain-containing proteins, involves a hydroxylation reaction requiring ferrous ion and $\alpha$-ketoglutarate as cofactors $(11,13)$.

$\mathrm{Kdm} 3 \mathrm{a}$ has been implicated in the regulation of gene expression during numerous biological functions, including spermatogenesis, metabolism, sex determination, androgen receptor-mediated transcription and cell differentiation (14-17). Aberrant expression of $k d m 3 a$ is associated with several malignancies, including colorectal cancer, breast cancer, hepatocellular carcinoma and hypoxia (18-21).

Multiple previous studies indicated the involvement of H3K9me2 demethylation in neural development (22-26). In human embryonic stem cells, $\mathrm{Kdm} 3 \mathrm{c}$ was reported to serve roles in the repression of neural differentiation and was necessary for the maintenance of miR-302 expression (22). miR-302 is an epigenetic regulator of pluripotency and neural differentiation (27). Furthermore, depletion of lysine demethylase 1a splice variant catalyzing demethylation of $\mathrm{H} 3 \mathrm{~K} 9 \mathrm{me} 1 / 2$, increases the level of $\mathrm{H} 3 \mathrm{~K} 9 \mathrm{me} 2$ at the target promoters associated with the impairment of neuronal differentiation (23). In addition, lysine demethylases $7 \mathrm{a}$ and $7 \mathrm{~b}$ which demethylate $\mathrm{H} 3 \mathrm{~K} 9 \mathrm{me} 1 / 2$ are also involved in the regulation of neural development $(24,25)$.

Xenopus laevis is a model used to investigate the mechanisms underlying neural development and differentiation $(28,29)$. The present study investigated the developmental functions of $\mathrm{Kdm} 3 \mathrm{a}$ and its effects on craniofacial and neural development during Xenopus embryogenesis. Whole-mount in situ hybridization (WISH) analysis revealed the expression of $k d m 3 a$ in the anterior regions of Xenopus embryos, including the retina, central nervous system (CNS) and branchial arches. Morpholino antisense oligonucleotide (MO)-mediated knockdown of $k d m 3 a$ resulted in defective craniofacial development and neural deformities. Furthermore, the results of WISH using neural crest-specific probes indicated that $\mathrm{Kdm} 3 \mathrm{a}$ regulated neural crest migration as $k d m 3 a$ morphant embryos exhibited decreased migration of neural crest cells compared with control MO-injected embryos. In addition, $k d m 3 a$ depletion altered the expression of neural markers and downregulated the expression of genes specific for mesoderm development, cell adhesion and metabolism. The results of the present study suggest that Kdm3a may serve important roles during Xenopus embryogenesis by regulating the expression of neural-specific genes.

\section{Materials and methods}

Xenopus husbandry and in vitro fertilization. Xenopus laevis (age, 3 years old; 20 females and 10 males) were obtained from the Korean Xenopus Resource Center for Research and housed with a 12 -h light/dark cycle at $18^{\circ} \mathrm{C}$ in containers built specifically according to the requirements for maintenance of laboratory organisms by the Institutional Review Board of Ulsan National Institute of Science and Technology (ethical approval no. UNISTACUC-16-14). To induce ovulation, Xenopus females were injected with 1,000 IU/animal of human chorionic gonadotropin (Dae Sung Microbiological Labs., Co., Ltd., Seoul, Korea) into the dorsal lymph sac in the evening. The following day, the abdomens of female frogs were gently squeezed and eggs were placed in $60-\mathrm{mm}$ petri dishes containing 1X Modified Barth's Saline (MBS; $88 \mathrm{mM}$ $\mathrm{NaCl}, 5 \mathrm{mM}$ Hepes, $2.5 \mathrm{mM} \mathrm{NaHCO}$, $1 \mathrm{mM} \mathrm{KCl,} 1 \mathrm{mM}$ $\mathrm{MgSO}_{4}$, and $0.7 \mathrm{mM} \mathrm{CaCl}$, $\mathrm{pH}$ 7.8). After washing three times with 0.1X MBS, eggs were fertilized using a suspension solution of sperm obtained from isolated testes of sacrificed males. Following successful fertilization, the embryos were swirled in a $2 \%$ L-cysteine solution (Sigma-Aldrich; Merck KGaA, Darmstadt, Germany) to remove the jelly coat and washed five times with $0.5 \mathrm{X}$ MBS. Live and healthy embryos were transferred to $0.5 \mathrm{X}$ MBS containing $2 \%$ Ficoll $^{\circledR}$ PM 400 (GE Healthcare Life Sciences, Little Chalfont, UK) while unfertilized eggs and non-viable embryos were removed by observation under a light microscope (magnification, $\mathrm{x} 1$ ).

Plasmids, mRNAs, MOs and microinjections of Xenopus embryos. A full-length $k d m 3 a$ cDNA clone (GeneBank ID: NM_001095502) was obtained from the American Type Culture Collection (Manassas, VA, USA). The amplification of Flag-tagged $k d m 3 a$ was performed by polymerase chain reaction (PCR), as previously described (30). The amplified fragment was sub-cloned into a pCS107 vector (Laboratory of Protein Dynamics and Signaling, National Cancer Institute, Frederick, MD, USA). Tagged $k d m 3 a$ was subsequently linearized with PvuII restriction endonucleases. SP6 mMessage mMachine kit (Ambion; Thermo Fisher Scientific, Inc., Waltham, MA, USA) was used to synthesize capped mRNAs for microinjections. The $k d m 3 a$ MOs and control MOs were synthesized by Gene Tools, LLC (Philomath, OR, USA; 5'-GTT CTC TTG CTG AGT GAG CAC CAT A-3'; Control MO, 5'-CCT CTT ACC TCA GTT ACA ATT TAT A-3'). Both blastomeres of two-cell staged embryos were microinjected with MOs (30 ng/blastomere) and/or mRNAs (1 ng) and incubated until the required stages (16, 22 and 32). For rescue experiments, a MO-resistant mRNA $\left(k d m 3 a^{*}\right)$ with seven point mutations in wobble codons followed by an ATG start codon (5'-ATG GTA CTG ACG CAA CAG GAA AAT-3') was synthesized. For tissue-specific expression of neural crest-specific markers, $k d m 3 a \mathrm{MO}$ was microinjected along with $\beta$-galactosidase (Laboratory of Protein Dynamics and Signaling, National Cancer Institute; $300 \mathrm{pg}$ ) into one of the two blastomeres of two-cell stage embryos.

Whole-mount in situ hybridization (WISH) and $\beta$-galactosidase staining. Xenopus embryos were collected at developmental stage 16, 22 and 32 and prefixed in MEMFA (4\% paraformaldehyde, 0.1 M MOPS pH 7.4, $1 \mathrm{mM} \mathrm{MgSO}_{4}$ and $2 \mathrm{mM}$ EGTA) for $2 \mathrm{~h}$ at room temperature. WISH was performed on fixed embryos by hybridization with neural crest-specific probes, including Twist-family bHLH transcription factor 1 (twist), snail family zinc finger 2 (snail2), transcription factor AP-2 $\alpha$ (tfap $2 a)$, early growth response 2 (egr 2$)$ forkhead box d3 (foxd3), myc proto-oncogene, bHLH transcription factor $(c-m y c)$, msh homeobox 1 ( $m s x l)$ and paired box 3 (pax3), as previously described (31). To distinguish the MO-injected side of the embryos, $\beta$-galactosidase staining was performed, as previously described (31). 
Table I. Primers used for qPCR.

Primer sequence $\left(5^{\prime} \rightarrow 3^{\prime}\right)$

\begin{tabular}{lll}
\multicolumn{1}{c}{ Gene } & \multicolumn{1}{c}{ Forward } & \multicolumn{1}{c}{ Reverse } \\
\hline$o d c$ & CAG CTA GCT GTG GTG TGG & CAA CAT GGA AAC TCA CAC C \\
$k d m 3 a$ & TCC TAC ACC TGC CTG CTC T & CAA GCT GGG CTG CAT TGG A \\
$a g 1$ & AAG GCC TGA AGA CCC TGG A & TGC GAG AGT CAG GGA TGG A \\
$h e s x l$ & AGC TTT CAC TAG GAG CCA GA & AGG TCC AAG GCT CTA TCA \\
$z i c 3$ & CAA CAG TGA GGA ACC TTC CA & GGG CTT TGT TAG TCT GTA GC \\
six3 & AAC ACG AGT CCA TCC TGC G & CCT GCT GGG GTT GGG GTA G \\
otx2 & GGA TGG ATT TGT TAC ATC CGT C & CAC TCT CCG AGC TCA CTT CCC \\
$n$ n-cam & ATG AGC AGA ATA ACA GGG AAG TGG A & CCT CGG GGACAT TGA CTC GGT GGT G \\
foxgl & GCG GGT ACC TTC TAA TAG TCA C & GGC TTG GCT GTG GTT CTG AAG G \\
\hline
\end{tabular}

$o d c$, ornithine decarboxylase; $k d m 3 a$, lysine demethylase 3a; Ag1, anterior gradient 1; hesx 1, HESX hmeobox1; zic3, ZIC family member 3; six3, SIX homeobox 3; otx2, orthodenticle homeobox 2; en2, engrailed homeobox 2; n-cam, neural cell adhesion molecule 1; foxg1, forkhead box G1 .

Quantitative (q)PCR. Total RNA extraction was performed by lysing the embryos in Isol-RNA lysis reagent (5 Prime GmbH, Hamburg, Germany) and cDNA was prepared using the first strand cDNA synthesis kit (Takara Bio, Inc., Otsu, Japan) at $65^{\circ} \mathrm{C}$ for $5 \mathrm{~min}$ followed by $42^{\circ} \mathrm{C}$ for $1 \mathrm{~h}$ and $95^{\circ} \mathrm{C}$ for $5 \mathrm{~min}$. qPCR reaction was performed using specific primers (Table I) and SYBR Premix Ex Taq, according to the manufacturer's protocol (Takara Bio, Inc.). Thermocycler was adjusted at $95^{\circ} \mathrm{C}$ for $30 \mathrm{sec}$ to ensure denaturation, annealing temperature was set at $55^{\circ} \mathrm{C}$ for $30 \mathrm{sec}$ followed by extension at $72^{\circ} \mathrm{C}$ for $1 \mathrm{~min}$ (30 cycles). The analysis was performed using StepOnePlus ${ }^{\mathrm{TM}}$ Real-Time PCR system (Applied Biosystems; Thermo Fisher Scientific, Inc.). The relative expression levels of target genes were analyzed using the $2^{-\Delta \Delta \mathrm{Cq}}$ method (32). All data are representative of at least three experiments. Primers were designed using Primer3 software (33) and ornithine decarboxylase was used as an internal control.

Animal cap assay. Xenopus embryos were microinjected with $k d m 3 a \mathrm{MO}$ and/or 2 ng of dominant negative bone morphogenetic protein 4 receptor ( $d n b r)$ mRNA (Laboratory of Protein Dynamics and Signaling, National Cancer Institute) which induced neural tissue formation from the prospective ectoderm. The animal caps were excised using forceps following removal of the chorion membrane from the vegetal side of late blastula (stage 8.5-9 embryos). Excised animal caps were cultured at $18^{\circ} \mathrm{C}$ in $0.5 \mathrm{X}$ MBS containing 2\% Ficoll PM 400 until developmental stage 16 of Xenopus embryogenesis (34).

Alcian blue staining. For alcian blue staining, Xenopus embryos were collected at the late tadpole stage (stage 43) and fixed in Bouin's solution for $2 \mathrm{~h}$ at room temperature. Following fixation, embryos were washed in $70 \%$ ethanol $+0.1 \% \mathrm{NH}_{4} \mathrm{OH}$ and stained with $0.05 \%$ Alcian Blue 8GX (Sigma-Aldrich; Merck $\mathrm{KGaA}$ ) in $5 \%$ acetic acid for $>2 \mathrm{~h}$ at room temperature. The embryos were subsequently washed in $5 \%$ acetic acid for $2 \mathrm{~h}$ at room temperature and cleared in $100 \%$ methanol. Following clearing, the embryos were kept in benzyl benzoate and benzyl alcohol at a ratio of 2:1, respectively.

Western blot analysis. The $k d m 3 a \mathrm{MO}$-injected embryos were treated with the lysis buffer $(137 \mathrm{mM} \mathrm{NaCl}, 20 \mathrm{mM}$ Tris- $\mathrm{HCl}$ $\mathrm{pH} 8.0,1 \%$ Nonidet-P40 and $10 \%$ glycerol) and $1 \mathrm{mM}$ phenylmethylsulfonyl fluoride (Amresco, LLC, Solon, OH, USA), $5 \mathrm{mM}$ sodium orthovanadate (Sigma-Aldrich; Merck KGaA) and $1 \mathrm{X}$ protease inhibitor mix (Roche Diagnostics, Basel, Switzerland), followed by heating of lysates at $95^{\circ} \mathrm{C}$ in loading buffer for $5 \mathrm{~min}$. Bradford assay was used for protein determination; lysates (40 $\mu \mathrm{g} /$ lane) were analyzed using SDS-PAGE (12\% gel) followed by transfer to nitrocellulose membranes. The membranes were blocked with skim milk $(5 \%)$ for $1 \mathrm{~h}$ at room temperature and proteins were detected with anti-Flag tag (cat. no. G188; Applied Biological Materials, Inc., Richmond, BC, Canada) for $1 \mathrm{~h}$ at room temperature. Subsequently, the membranes were incubated with goat anti-mouse (cat. no. sc2005; Santa Cruz Biotechnology, Inc., Dallas, TX, USA) and goat anti-rabbit (cat.no. sc2004; Santa Cruz Biotechnology, Inc.) horseradish peroxidase-conjugated antibodies by incubating for $1 \mathrm{~h}$ at room temperature. Primary and secondary antibodies were used at a dilution of 1:1,000. The immunoreactive bands were detected using an enhanced chemiluminescence kit (HyGLO ${ }^{\mathrm{TM}}$ kit; Denville Scientific, Inc., South Plainfield, NJ, USA). Histone H3 (cat. no. ab1791; Abcam, Cambridge, UK) antibody is used as a loading control.

RNA sequencing. Total RNA was harvested from each sample (3 embryos/sample) at tadpole stage (stage 32) and RNA sequencing library was constructed according to manufacturer's protocol (TruSeq RNA Sample Prep kit v.2; cat. nos. RS-122-2001 and RS-122-2002; Illumina, Inc., San Diego, CA, USA) using polyA enrichment. For estimation of mRNA abundance, the reads were mapped to the Xenopus laevis cDNA sequences from the genome project consortium (35) using BWA software (v.0.7.15) (36), and the 
significant differential expression of genes was estimated using edgeR software (v.3.3.1). Genes exhibiting $>4$-fold change and false discovery rate $<0.01$ were considered significantly differentially expressed. To determine biological processes in which the differentially expressed genes were enriched, Fisher's test provided by the PANTHER database (release 20171205) (37) was used with human orthologous genes based on the best-hit results using BLASTP search (38). RNA sequencing raw data are available at the National Center for Biotechnology Information Gene Expression Omnibus database (accession no. GSE117754).

Statistical analysis. Image J software (v.1.45; National Institutes of Health, Bethesda, MD, USA) was used to analyze the data from the WISH and PCR analyses, and the results are presented as the mean \pm standard error of the mean. Results obtained from three independent experiments. The level of significance was calculated using an unpaired t-test or one-way analysis of variance followed by Tukey's post hoc test using GraphPad Prism software (v.7; GraphPad Software, Inc., La Jolla, CA, USA). P $<0.05$ was considered to indicate a statistically significant difference.

\section{Results}

$k d m 3 a$ is expressed at the anterior regions during Xenopus embryogenesis. To study the function of Kdm3a during Xenopus embryonic development, the present study investigated the expression pattern of $k d m 3 a$ in early Xenopus embryos. qPCR analysis indicated that $k d m 3 a$ was expressed at early stages of embryogenesis, ranging from the egg cell to the tadpole stage of development (Fig. 1A). However, markedly increased level of $k d m 3 a$ expression was observed at the neurula stage (stage 17; Fig. 1A). The spatial expression of $k d m 3 a$ was analyzed using WISH, and $k d m 3 a$ mRNA localized to the CNS during the neurula stage of embryogenesis (stage 16; Fig. 1B). Furthermore, $k d m 3 a$ was expressed at the anterior region, including the eye and the CNS at stage 22 (early tailbud stage), and was observed in the retina, otic vesicle and branchial arches at stage 32 (late tailbud stage) of Xenopus embryogenesis (Fig. 1B). The results of qPCR and WISH analysis indicate that $k d m 3 a$ may serve roles during Xenopus embryogenesis and regulate neurogenesis during embryonic development.

Kdm3a knockdown arrests craniofacial formation during Xenopus embryogenesis. To investigate the physiological functions of Kdm3a during Xenopus embryogenesis, loss-of-function experiments were performed using $k d m 3 a$ MO to inhibit $k d m 3 a$ translation. Embryos at the two-cell stage were injected with $k d m 3 a \mathrm{MO}$ to repress $k d m 3 a$ expression.

Embryos injected with the $k d m 3 a \mathrm{MO}$ exhibited malformed phenotypes, including defective head formation and markedly smaller eyes compared with the control MO-injected embryos (Fig. 2A). Anterior defects were identified in $>90 \%$ of $k d m 3 a$ morphants (Fig. 2B). Furthermore, abnormal pigmentation was observed among $k d m 3 a$ morphants (Fig. 2C). Alcian blue staining was performed to visualize the effects of $k d m 3 a$ MOs on craniofacial development. $k d m 3 a$ morphants exhibited markedly smaller cartilage compared with cartilage formation in control embryos (Fig. 2C).

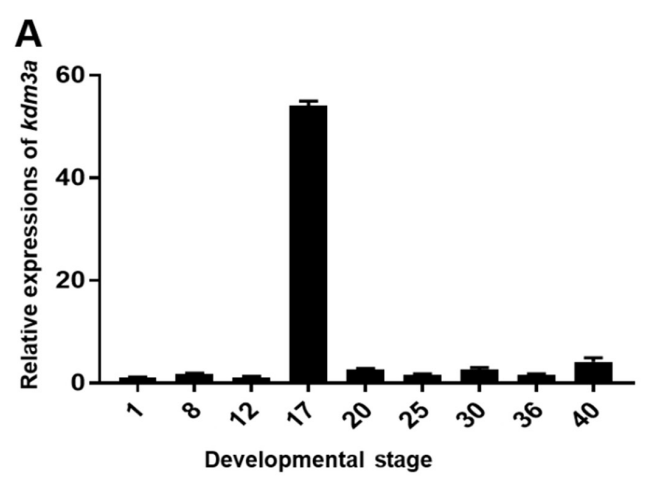

B

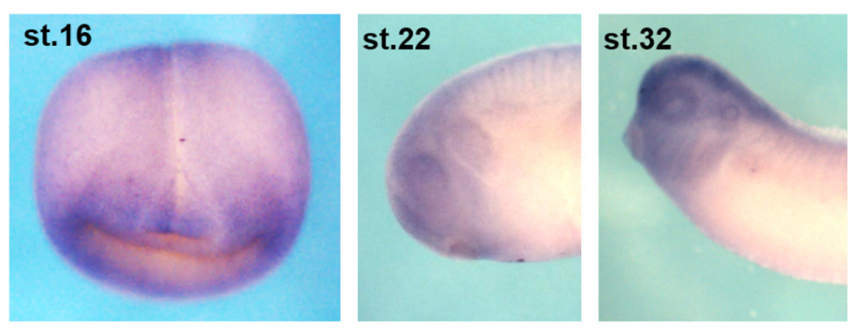

Figure 1. Expression patterns of $k d m 3 a$ during Xenopus embryogenesis. (A) Expression pattern confirmed by quantitative polymerase chain reaction. Ornithine decarboxylase served as the loading control. (B) Spatial expression pattern of $k d m 3 a$ was analyzed by whole-mount in situ hybridization. Xenopus embryos were collected at stage 16 (neurula stage), 22 and 32 (tail bud stage) and analyzed under a light microscope (magnification, x2.5). st., stage; kdm3a, lysine demethylase 3a.

To confirm whether the malformed phenotypes were specifically induced by the depletion of $k d m 3 a$, rescue experiments were performed by microinjecting $k d m 3 a^{*}$ into two-cell stage embryos. Injection of $k d m 3 a^{*}$ recovered the malformed phenotypes observed in $k d m 3 a$ morphants (Fig. 2D). This validation experiment confirmed that the defects observed in $k d m 3 a$ morphants were caused by $k d m 3 a$ knockdown during Xenopus embryogenesis. Furthermore, western blot analysis was used to examine protein expression of $k d m 3 a$. No $k d m 3 a$ expression was observed in the group treated with MO, confirming the efficiency of knockdown (Fig. 2E). In addition, protein expression in embryos injected with $k d m 3 a^{*}$ or $k d m 3 a^{*}+\mathrm{MO}$, verified that MO did not bind to $k d m 3 a^{*}$ (Fig. 2E). These results suggested that the malformed phenotypes including defective craniofacial formation were caused by the loss of $k d m 3 a$, validating the role of this gene during Xenopus embryonic development.

Depletion of kdm3a affects neural crest migration during Xenopus embryonic development. The cells of neural crest emanate from neural tube immediately after its closure, and subsequently, migrate to specific regions of the embryo where they differentiate to the peripheral nervous system, facial skeleton cells and pigment cells $(39,40)$. As described above, $k d m 3 a$ morphants exhibited defects in craniofacial formation, abnormal head development and dense pigmentation (Fig. 2C), and $k d m 3 a$ expression was observed at the neural plate during the neural stage of development (Fig. 1B). The present study further investigated whether $k d m 3 a$ may affect neural crest migration. 
A
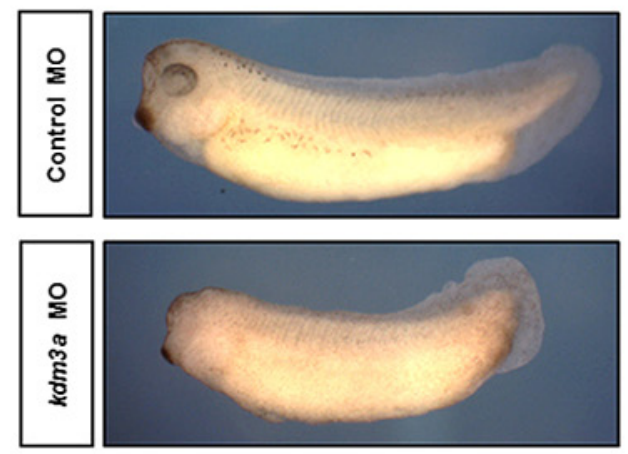

B

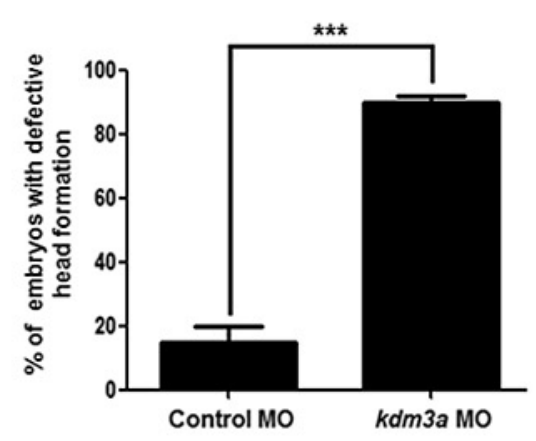

C

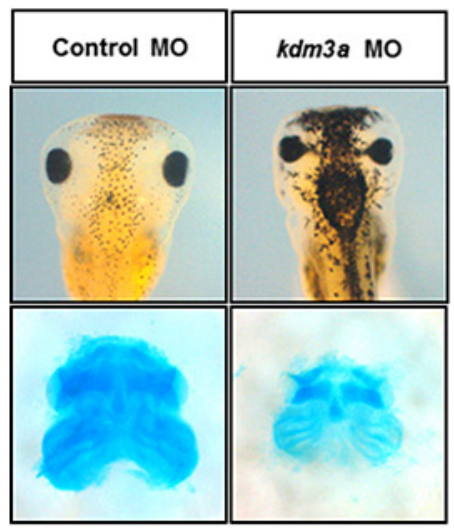

D

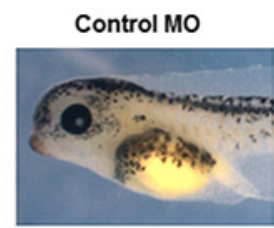

$k d m 3 a \mathrm{MO}$

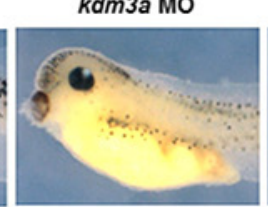

$k d m 3 a \mathrm{MO}+k d m 3 a^{*}$

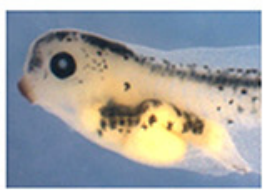

E

Figure 2. Depletion of $k d m 3 a$ results in defective head formation and reduces cartilage formation. (A) $k d m 3 a$ MOs were microinjected into two-cell stage embryos. Embryos were collected at stage 32 (late tailbud). (B) Quantitative analysis of embryos exhibiting defective head formation. (C) Alcian blue staining to visualize cartilage formation. Embryos were fixed at stage 43 (late tailbud). (D) Rescue experiments investigating malformed phenotypes induced by $k d m 3 a$ depletion using microinjection of $k d m 3 a *$ together with $k d m 3 a$ MO. (E) Western blot analysis for $k d m 3 a$. H3 was used as the loading control. Embryos were analyzed under a light microscope (magnification, x2.5). ${ }^{* * *} \mathrm{P}<0.001$ as indicated. Kdm3a, lysine demethylase $3 \mathrm{a}$; $k d m 3 a^{*}, \mathrm{MO}-\mathrm{resistant}$ mRNA; MO, morpholino antisense oligonucleotide; $\mathrm{H} 3$, histone $\mathrm{H} 3$.

To examine the role of $\mathrm{Kdm} 3 \mathrm{a}$ in neural crest migration, $k d m 3 a \mathrm{MO}$ and $\beta$-galactosidase mRNA were injected into one blastomere of two-cell stage embryos. The injected sides of the embryos were visualized by $\beta$-galactosidase assays and WISH analysis was performed using neural crest marker genes. twist, snail2, tfap $2 a$, egr 2 and foxd 3 are widely used neural crest marker genes $(41,42)$. WISH expression patterns of twist (stage 32; late tailbud), and snail2, foxd3 and tfap2a (all at stage 20; neurula) indicated that injection with $k d m 3 a \mathrm{MO}$ significantly reduced neural crest migration on the injected side of the embryos (Fig. 3). In addition, $k d m 3 a$ MO-injected sides of the embryos exhibited abnormal expression patterns of egr2- $a$, a hindbrain-specific marker; however, normal development of rhombomere 3 and 5, and neural crest was observed in the uninjected sides of the embryos (Fig. 3). These genes serve important roles during development of progenitor cells of neural crest into bona fide neural crest cells (43). Furthermore, twist is involved in neural crest cell migration as a repressor of transcription (44). Injected embryos exhibited abnormal expression patterns of twist, snail2 and foxd3, compared with the uninjected control (Fig. 3B).

It has been previously demonstrated that $\mathrm{Kdm} 3 \mathrm{a}$ may serve important roles in primary neuron formation; however, it was not required for neural induction (45). To verify these previous results, the present study observed the expression patterns of early markers of neural crest progenitor cells, including $c$-myc (46), msxl and pax3 (47). Expression of $c$-myc was not affected by $k d m 3 a$ knockdown (Fig. 3). Expression patterns of $m s x 1$ and pax3-neural crest induction markers $(47,48)$ remained unaltered on the $k d m 3 a$ MO-injected side (Fig. 3). Therefore,
WISH analysis results together with the $\beta$-galactosidase assay demonstrated that depletion of $k d m 3 a$ may affect the migration of neural crest cells by disturbing the expression of neural crest specifiers. Since facial cartilage is formed by the cranial neural crest and trunk neural crest cells differentiate into pigment cells $(49,50)$; therefore, it may be hypothesized that the reduced size of cartilage and abnormal pigmentation in $k d m 3 a$ morphants resulted from abnormal neural crest migration.

kdm3a knockdown alters the expression of neural-specific genes. $\mathrm{Kdm} 3 \mathrm{a}$ serves essential roles in primary neuron formation and its depletion downregulates the expression of neural-differentiation associated genes (45). Therefore, the present study aimed to investigate the effect of Kdm3a on neural-specific (anterior, posterior and pan) genes. For this purpose, $k d m 3 a \mathrm{MO}$ and $d n b r$ mRNA were co-injected into the animal pole region of two-cell stage embryos. Animal caps were excised from the microinjected embryos at blastula stage (stage 8.5-9) and cultured until neurula stage (stage 16). Xenopus animal caps, equivalent to embryonic stem cells, develop into the ectoderm, however, these cells are pluripotent and can differentiate into neural, mesodermal and endodermal tissues depending on the level and type of specific inducers $(34,51) . d n b r$ induces the formation of neural tissue from animal cap explants by inhibiting bone morphogenetic protein (BMP) signaling during early development of Xenopus and increasing expression of neurogenesis-specific genes (34).

Gene expression levels in animal caps dissected from $k d m 3 a$ MO and $d n b r$ mRNA co-injected embryos were analyzed 


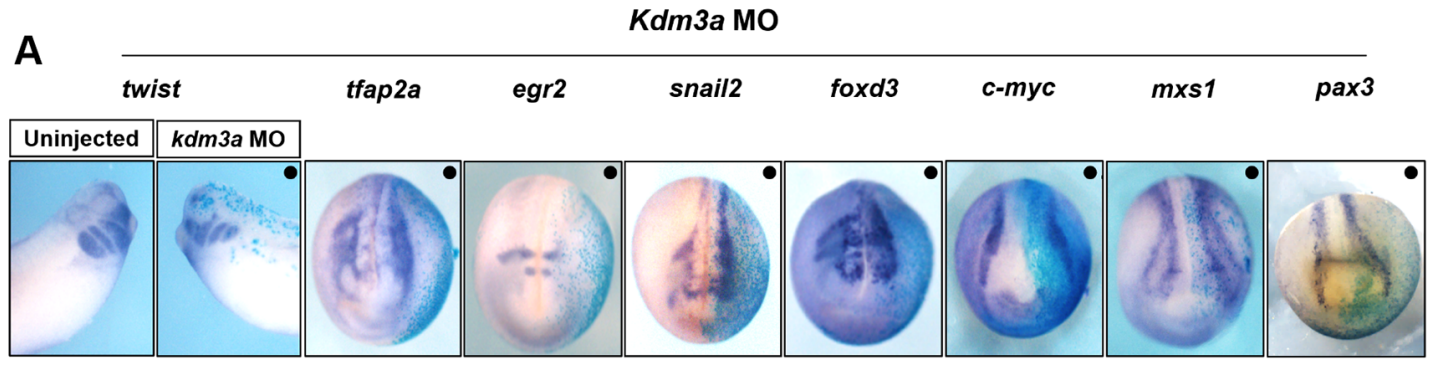

B

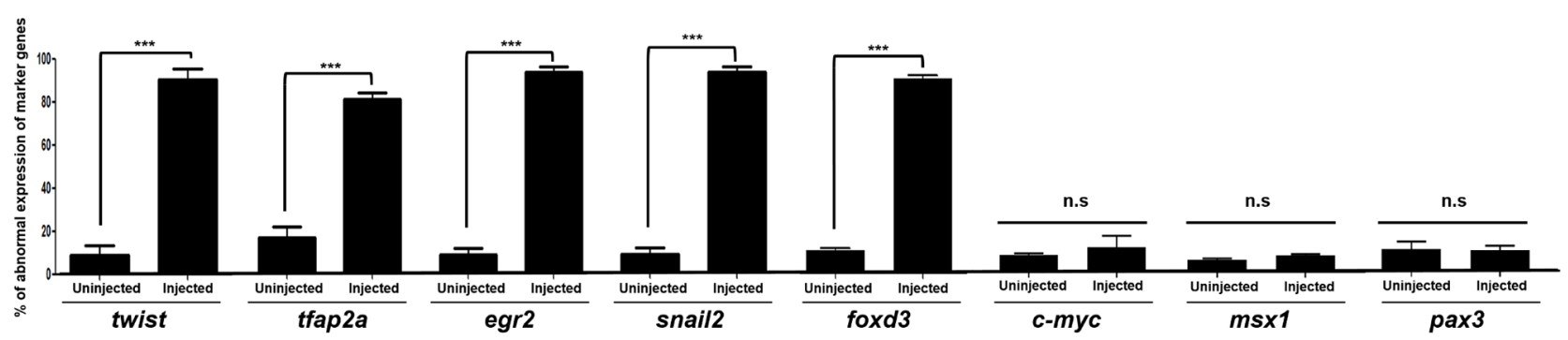

Figure 3. Kdm3a is required for neural crest migration by regulating the expression of neural crest specifiers. (A) $k d m 3 a \mathrm{MO}$ and $\beta$-galactosidase mRNA were co-injected into one blastomere of two-cell stage embryos. Embryos were harvested at stage 16, 20 (neurula), and stage 32 (late tailbud). $k d m 3 a$ depletion led to defective neural crest migration as indicated by the expression patterns of $t$ wist (stage 32; late tailbud), tfap2a, egr2, snail2, foxd3 (stage 20; neurula stage), $c$-myc, msx1 and pax3 (stage 16; neurula stage). Injected sides of the embryos exhibited reduced neural crest cell migration compared with the uninjected sides of the embryos. The black dot $(\bullet)$ represents the injected side. Embryos were analyzed under a light microscope (magnification, $\mathrm{x} 2.5$ ). (B) Expression patterns of neural crest specifiers, including twist, tfap2a, egr2, snail2, foxd3, c-myc, msx1 and pax3 induced by kdm3a knockdown. Data are presented as the mean \pm standard error of the mean. ${ }^{* * * *} \mathrm{P}<0.001$, as indicated. n.s, not significant; twist, twist-family bHLH transcription factor 1 ; snail 2 , snail family zinc finger 2; tfap $2 a$, transcription factor AP-2 $\alpha$; egr2, early growth response 2 ; foxd 3 , forkhead box d3; $c$-myc, myc proto-oncogene bHLH transcription factor; msxl, msh homeobox 1; pax3, paired box 3; kdm3a, lysine demethylase 3a; MO, morpholino antisense oligonucleotide.

by qPCR to determine the neural marker gene expression. Depletion of $k d m 3 a$ led to the inhibition of expression of cement gland marker, anterior gradient 1 (agl) (52), anterior markers, orthodenticle homeobox 2 (otx2), HESX hmeobox1 (hesx1) and forkhead box G1 (foxgl) (53), pan-neural marker, neural cell adhesion molecule 1 ( $n$-cam) (48), and retina marker, SIX homeobox 3 (six3) (54), consistent with the $k d m 3 a$ morphant phenotype (Fig. 4). By contrast, the expression levels of neural crest marker, ZIC family member 3 and posterior neural marker, engrailed homeobox 2 (55), were not suppressed by the knockdown of $k d m 3 a$ (Fig. 4). Our qPCR data for neural marker gene expression are consistent with the previously published data (45). Furthermore, the present study indicated that $k d m 3 a$ depletion inhibited the expression of six3. It may be hypothesized that $k d m 3 a$ serves a role in the expression of retina specific markers at the early stage of embryonic development.

The present study further conducted a transcriptomics analysis to elucidate the role of $\mathrm{Kdm} 3 \mathrm{a}$ during Xenopus embryogenesis. Transcriptomics analysis demonstrated that $k d m 3 a$ is important for metabolism, cell-cell adhesion and mesoderm development during Xenopus embryogenesis (Fig. 5). Knockdown of $k d m 3 a$ led to downregulation of a number of genes involved in mesoderm patterning and development, including fibroblast growth factor 13 ( $f g f 13)$, signal transducer and activator of transcription 3 gene 1 (stat3) and T-box 5 (tbx5) (56). Furthermore, the depletion of $k d m 3 a$ induced the downregulation of multiple genes involved in mesoderm induction and patterning (Fig. 5). Knockdown of $k d m 3 a$ suppressed the expression of genes involved in cell-cell adhesion, including angiopoietin 2 (angpt2), cadherin 5 (cdh5) and fibrinogen $\beta$ chain (fgb) (Fig. 5).
qPCR analysis using animal caps and the transcriptomics data indicated that $k d m 3 a$ depletion altered the expression of various genes involved in fundamental developmental processes. Therefore, $\mathrm{Kdm} 3 \mathrm{a}$ may serve an important role in normal embryonic development of Xenopus laevis.

\section{Discussion}

Epigenetic regulator $\mathrm{Kdm} 3 \mathrm{a}$ catalyzes demethylation of $\mathrm{H} 3 \mathrm{~K} 9 \mathrm{me} 1 / 2$, and is involved in the maintenance of the histone code (15). It has been reported that this demethylase is involved in numerous biological and pathological processes, including germ cell development, sex determination, metabolism, stem cell differentiation, stem cell self-renewal and cancer epigenetics $(2,57)$. In the present study, the expression pattern and physiological functions of $\mathrm{Kdm} 3 \mathrm{a}$ were examined during embryonic development of Xenopus.

Appropriately regulated gene expression is necessary in the process of embryonic growth and development. Maternal and zygotic $k d m 3 a$ was expressed during early stages of Xenopus embryonic development, until the tadpole stage of embryogenesis. The $k d m 3 a$ expression pattern observed in the present study is consistent with a previously conducted study (35). The qPCR analysis demonstrated that expression levels of $k d m 3 a$ varied across developmental stages and were highest during the neurula stage (stage 17) of embryogenesis. $k d m 3 a$ was expressed in the CNS during the neurula stage of embryonic development. The expression of $k d m 3 a$ was observed in the anterior region, including the eye and the CNS at stage 22 (early tailbud), retina, otic vesicle, and branchial arches at stage 32 (late tailbud) of Xenopus embryogenesis. Microinjection of $k d m 3 a$ MO into the 

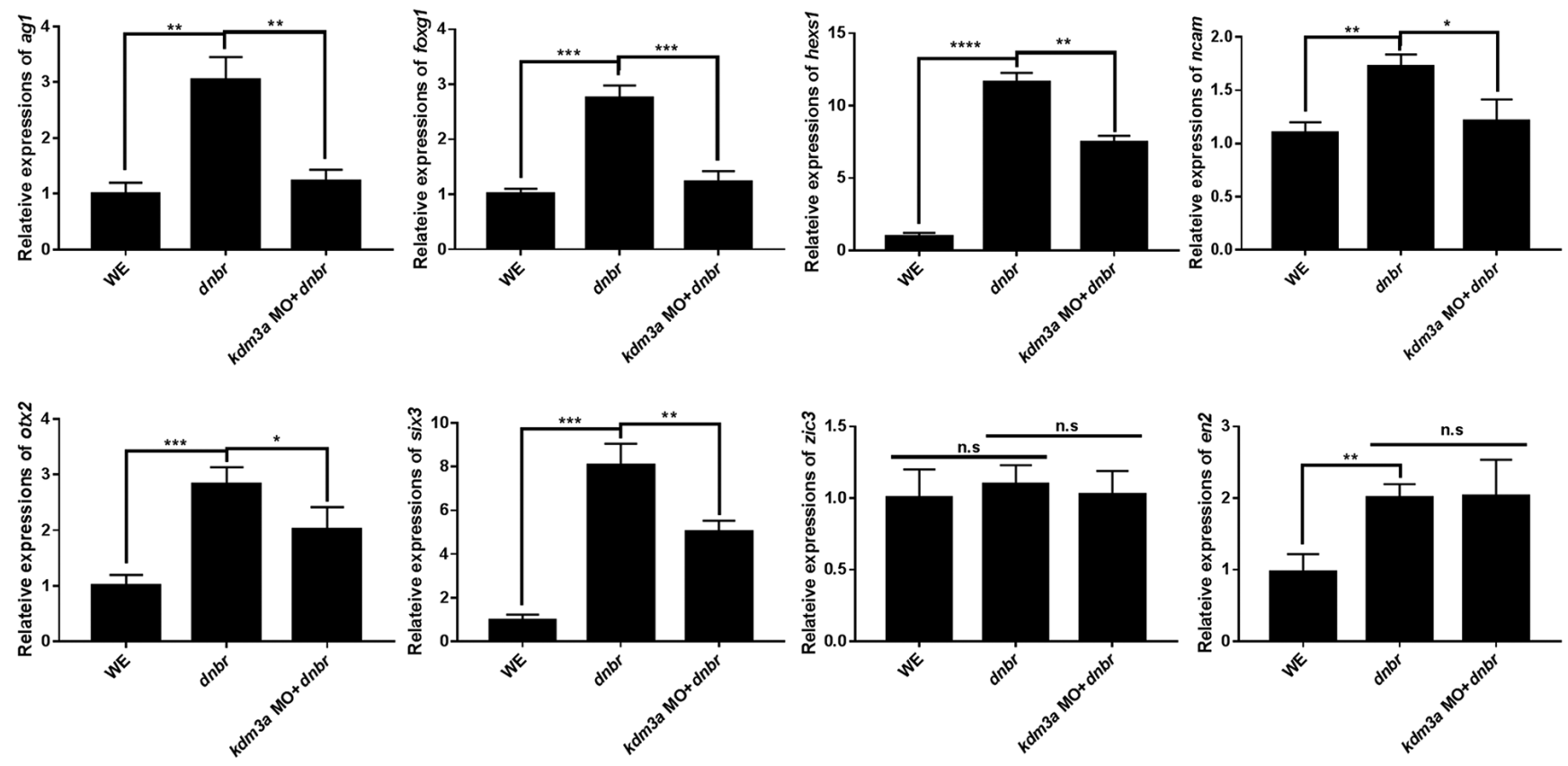

Figure 4. Kdm3a affects the expression of neural specific markers. Embryos were co-injected with $k d m 3 a \mathrm{MO}$ and $d n b r$ mRNA into the animal pole region of two-cell stage embryos. Animal caps were dissected from injected embryos at stage 8.5-9 (blastula) and incubated in 0.5X MBS. Animal caps were collected at stage 16 (neurula) and processed for reverse transcription-quantitative polymerase chain reaction using standard methods to detect the expression levels of hesxl, ag1, foxg1, otx 2, $n$-cam, zic3, six 3 and en2. odc was used as the loading control. $\mathrm{P}<0.05,{ }^{* * *} \mathrm{P}<0.01$ and ${ }^{* * * *} \mathrm{P}<0.001$, as indicated. n.s, not significant; kdm3a, lysine demethylase 3a; MO, morpholino antisense oligonucleotide; $d n b r$, dominant negative bone morphogenetic protein 4 receptor; WE, whole embryos; ag1, anterior gradient 1; odc, ornithine decarboxylase; hesxl, HESX hmeobox 1; zic3, ZIC family member 3; six3, SIX homeobox 3; otx2, orthodenticle homeobox 2; en2, engrailed homeobox 2; $n$-cam, neural cell adhesion molecule 1; foxg1, forkhead box G1.

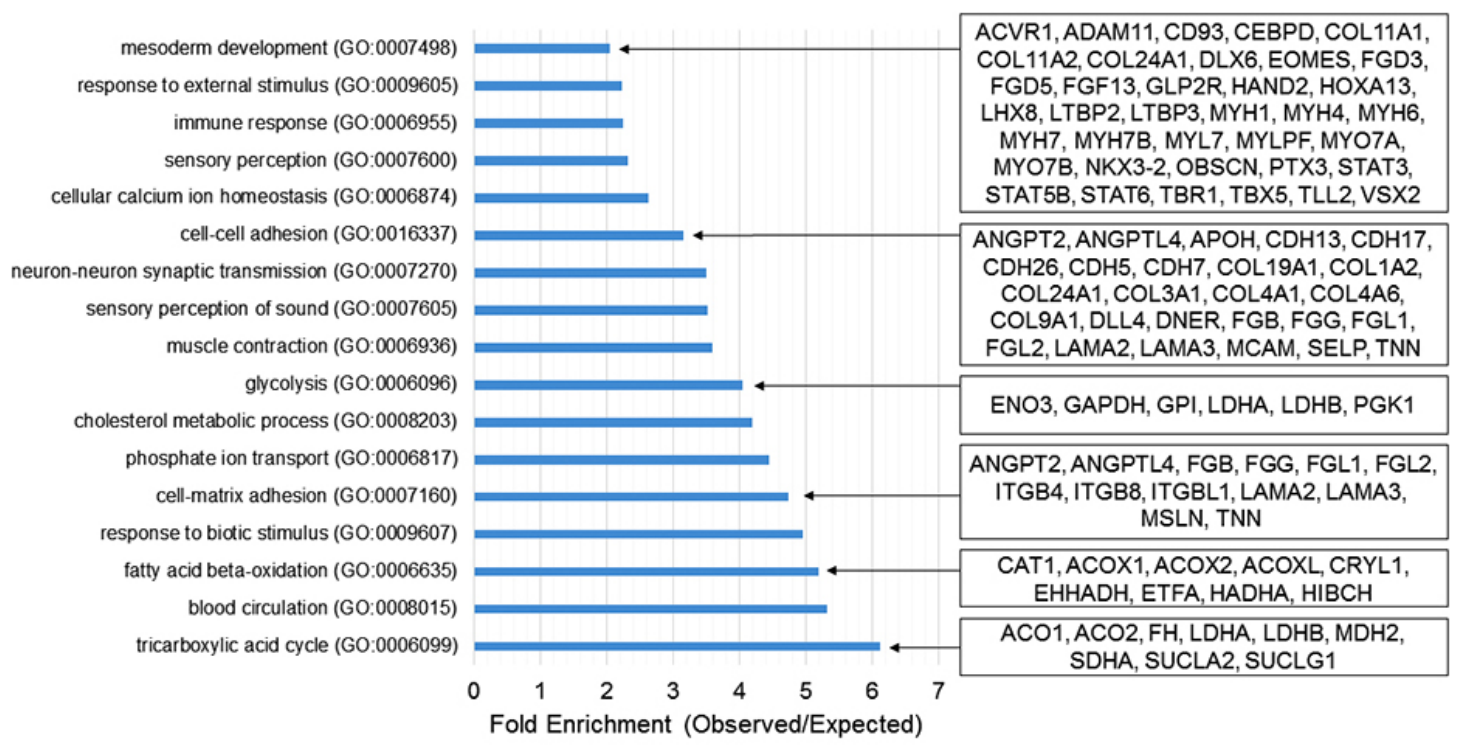

Figure 5. Metabolism, cell adhesion and mesoderm development. GO terms are affected by $k d m 3 a$ knockdown. RNA-sequencing was performed and the enriched biological processes were analyzed for significantly downregulated genes ( $>4$-fold change; FDR $<0.01$ ) following $k d m 3 a$ knockdown. GO, Gene Ontology; kdm3a, lysine demethylase 3a.

two-cell stage Xenopus embryos induced phenotypic abnormalities, including reduced head size and smaller eyes, compared with control MO-injected embryos. Craniofacial development of $k d m 3 a$ morphants was also affected, exhibiting markedly reduced cartilage and abnormal pigmentation, compared with the control embryos. Rescue experiments confirmed the role of Kdm3a during Xenopus embryogenesis. All malformed phenotypes of $k d m 3 a$ morphants were rescued following co-injection of $k d m 3 a \mathrm{MO}$ and $k d m 3 a^{*}$. These results indicate that $\mathrm{Kdm} 3 \mathrm{a}$ may serve important roles during neural crest migration of Xenopus embryos.

Jmjc domain-containing demethylases are involved in neural development. $\mathrm{Kdm} 7$ is necessary for brain formation through its interaction with a BMP antagonist, the follistatin gene locus (26). Furthermore, Kdm6b is specific for demethylation of H3K27me3 and is involved in neural development (58). 
Based on the previous results regarding $\mathrm{Kdm} 3 \mathrm{a}$ and its expression at the neurula stage (45), the present study investigated the physiological effect of Kdm3a during neural development. Injection of $k d m 3 a \mathrm{MO}$ altered the expression of neural crest specifiers. The WISH analysis indicated markedly altered expression of neural crest specifiers as a result of $k d m 3 a$ knockdown. These results indicated that $k d m 3 a$ depletion may be associated with impaired neural crest migration and may be required for facial cartilage formation.

$\mathrm{Kdm} 3 \mathrm{a}$ serves a role in primary neuron formation and is known to regulate the expression of neuronal differentiation-associated genes; however the depletion of $k d m 3 a$ exhibits no effect on the expression of neural stem progenitor markers pax6 and sox3 (45). The results of the present study supported these previously published data and indicated that $\mathrm{Kdm} 3 \mathrm{a}$ may not be involved in the induction of neural crest cells, as demonstrated by the expression levels of early markers of neural progenitor cells, including $c-m y c, m s x l$ and pax3.

The present study analyzed the effect of $\mathrm{Kdm} 3 \mathrm{a}$ on neural marker gene expression in animal caps of $d n b r$-injected embryos. Loss-of-function of $k d m 3 a$ resulted in reduced expression of anterior neural markers hesxl, ot 2 and foxgl, and pan-neural marker $n$-cam, suggesting that $k d m 3 a$ may serve a role in the neural development during Xenopus embryogenesis. Transcriptomics data analysis of $k d m 3 a$ morphants indicated that $k d m 3 a$ may be required for mesoderm formation. The mesoderm is one of the earliest germinal layers and is essential for normal development of the skeletal system, muscular system and a major part of the neural system (59). Knockdown of $k d m 3 a$ downregulated the expression of $f g f 13$, stat 3 and $t b x 5$, and other genes associated with mesoderm development. The expression levels of angpt2, $c d h 5, f g b$ and other genes involved in cell-cell adhesion were also suppressed in $k d m 3 a$ morphants.

In conclusion, the present study indicated that Kdm3a, an epigenetic modifier associated with histone code maintenance, is physiologically relevant as a regulator of neurogenesis during Xenopus embryonic development. Additional investigation is required to determine the interaction between this epigenetic regulator and developmental pathways, which may provide novel methods for the treatment of developmental disorders.

\section{Acknowledgements}

Not applicable.

\section{Funding}

This study was supported by grants from the National Research Foundation of Korea (grant no. NRF-2015R1A2A1A10053265) and the Ministry of Science, ICT and Future Planning, the Republic of Korea (grant no. 2015R1A4A1042271).

\section{Availability of data and materials}

The datasets used and/or analyzed during the present study are available from the corresponding author on reasonable request. RNA sequencing raw data are available at the National Center for Biotechnology Information Gene Expression Omnibus database (accession no. GSE117754).

\section{Authors' contributions}

HKL, TI, CK and YK performed the experiments. HKL, TI, CK, JWP, OSK, BSK, DSL and HSL performed the data analysis and wrote the manuscript. TK, TJP and HSL designed the study, interpreted the results and critically analyzed the manuscript.

\section{Ethics approval and consent to participate}

Experiments were conducted according to the guidelines of the Animal Care and Use Committee consistent with international laws and policies (National Institute of Health Guide for the Care and Use of Laboratory Animals; NIH publication no. 85-23, 1985). The Institutional Review Board of Ulsan National Institute of Science and Technology in Korea approved the experimental use of amphibians (approval no. UNISTACUC-16-14). All members of the research group were trained for the appropriate care and use of experimental organisms. There were no unexpected cases of mortality of adult Xenopus during the present study.

\section{Patients consent for publication}

Not applicable.

\section{Competing interests}

The authors declare that they have no competing interests.

\section{References}

1. Meissner A: Epigenetic modifications in pluripotent and differentiated cells. Nat Biotechnol 28: 1079-1088, 2010.

2. Mohn F and Schübeler D: Genetics and epigenetics: Stability and plasticity during cellular differentiation. Trends Genet 25: 129-136, 2009.

3. Mattout A and Meshorer E: Chromatin plasticity and genome organization in pluripotent embryonic stem cells. Curr Opin Cell Biol 22: 334-341, 2010.

4. Jenuwein T: The epigenetic magic of histone lysine methylation. FEBS J 273: 3121-3135, 2006.

5. Black JC, Van Rechem C and Whetstine JR: Histone lysine methylation dynamics: Establishment, regulation, and biological impact. Mol Cell 48: 491-507, 2012.

6. Bernstein BE, Mikkelsen TS, Xie X, Kamal M, Huebert DJ, Cuff J, Fry B, Meissner A, Wernig M, Plath K, et al: A bivalent chromatin structure marks key developmental genes in embryonic stem cells. Cell 125: 315-326, 2006.

7. Tachibana M, Sugimoto K, Nozaki M, Ueda J, Ohta T, Ohki M, Fukuda M, Takeda N, Niida H, Kato H, et al: G9a histone methyltransferase plays a dominant role in euchromatic histone $\mathrm{H} 3$ lysine 9 methylation and is essential for early embryogenesis. Genes Dev 16: 1779-1791, 2002.

8. Klose RJ, Kallin EM and Zhang Y: JmjC-domain-containing proteins and histone demethylation. Nat Rev Genet 7: 715-727, 2006.

9. Fodor BD, Kubicek S, Yonezawa M, O'Sullivan RJ, Sengupta R, Perez-Burgos L, Opravil S, Mechtler K, Schotta G and Jenuwein T: Jmjd2b antagonizes H3K9 trimethylation at pericentric heterochromatin in mammalian cells. Genes Dev 20: 1557-1562, 2006

10. Wissmann M, Yin N, Müller JM, Greschik H, Fodor BD, Jenuwein T, Vogler C, Schneider R, Günther T, Buettner R, et al: Cooperative demethylation by JMJD2C and LSD1 promotes androgen receptor-dependent gene expression. Nat Cell Biol 9: 347-353, 2007.

11. Yamane K, Toumazou C, Tsukada Y, Erdjument-Bromage H, Tempst P, Wong J and Zhang Y: JHDM2A, a JmjC-containing H3K9 demethylase, facilitates transcription activation by androgen receptor. Cell 125: 483-495, 2006. 
12. Knebel J, De Haro L and Janknecht R: Repression of transcription by TSGA/Jmjdla, a novel interaction partner of the ETS protein ER71. J Cell Biochem 99: 319-329, 2006.

13. Cai C, Yuan X and Balk SP: Androgen receptor epigenetics. Transl Androl Urol 2: 148-157, 2013.

14. Inagaki T, Tachibana M, Magoori K, Kudo H, Tanaka T, Okamura M, Naito M, Kodama T, Shinkai Y and Sakai J: Obesity and metabolic syndrome in histone demethylase JHDM2adeficient mice. Genes Cells 14: 991-1001, 2009.

15. Kuroki S, Matoba S, Akiyoshi M, Matsumura Y, Miyachi H, Mise N, Abe K, Ogura A, Wilhelm D, Koopman P, et al: Epigenetic regulation of mouse sex determination by the histone demethylase Jmjd1a. Science 341: 1106-1109, 2013.

16. Okada Y, Scott G, Ray MK, Mishina Y and Zhang Y: Histone demethylase JHDM2A is critical for Tnp1 and Prm1 transcription and spermatogenesis. Nature 450: 119-123, 2007.

17. Tateishi K, Okada Y, Kallin EM and Zhang Y: Role of Jhdm2a in regulating metabolic gene expression and obesity resistance. Nature 458: 757-761, 2009.

18. Beyer S, Kristensen MM, Jensen KS, Johansen JV and Staller P: The histone demethylases JMJD1A and JMJD2B are transcriptional targets of hypoxia-inducible factor HIF. J Biol Chem 283 36542-36552, 2008

19. Ueda J, Ho JC, Lee KL, Kitajima S, Yang H, Sun W, Fukuhara N, Zaiden N, Chan SL, Tachibana M, et al: The hypoxia-inducible epigenetic regulators Jmjdla and G9a provide a mechanistic link between angiogenesis and tumor growth. Mol Cell Biol 34: 3702-3720, 2014

20. Wade MA, Jones D, Wilson L, Stockley J, Coffey K, Robson CN and Gaughan L: The histone demethylase enzyme KDM3A is a key estrogen receptor regulator in breast cancer. Nucleic Acids Res 43: 196-207, 2015.

21. Yamada D, Kobayashi S, Yamamoto H, Tomimaru Y, Noda T, Uemura M, Wada H, Marubashi S, Eguchi H, Tanemura M, et al: Role of the hypoxia-related gene, JMJD1A, in hepatocellular carcinoma: Clinical impact on recurrence after hepatic resection. Ann Surg Oncol 19 (Suppl 3): S355-S364, 2012.

22. Wang J, Park JW, Drissi H, Wang X and Xu RH: Epigenetic regulation of miR-302 by JMJD1C inhibits neural differentiation of human embryonic stem cells. J Biol Chem 289: 2384-2395, 2014.

23. Laurent B, Ruitu L, Murn J, Hempel K, Ferrao R, Xiang Y, Liu S, Garcia BA, Wu H, Wu F, et al: A specific LSD1/KDM1A isoform regulates neuronal differentiation through $\mathrm{H} 3 \mathrm{~K} 9$ demethylation. Mol Cell 57: 957-970, 2015

24. Huang C, Chen J, Zhang T, Zhu Q, Xiang Y, Chen CD and Jing N: The dual histone demethylase KDM7A promotes neural induction in early chick embryos. Dev Dyn 239: 3350-3357, 2010

25. Huang C, Xiang Y, Wang Y, Li X, Xu L, Zhu Z, Zhang T, Zhu Q, Zhang K, Jing N, et al: Dual-specificity histone demethylase KIAA1718 (KDM7A) regulates neural differentiation through FGF4. Cell Res 20: 154-165, 2010.

26. Tsukada Y, Ishitani T and Nakayama KI: KDM7 is a dual demethylase for histone H3 Lys 9 and Lys 27 and functions in brain development. Genes Dev 24: 432-437, 2010.

27. Lipchina I, Studer L and Betel D: The expanding role of miR-302-367 in pluripotency and reprogramming. Cell Cycle 11: $1517-1523,2012$

28. Hemmati-Brivanlou A, Kelly OG and Melton DA: Follistatin, an antagonist of activin, is expressed in the Spemann organizer and displays direct neuralizing activity. Cell 77: 283-295, 1994

29. Sasai Y, Lu B, Steinbeisser H, Geissert D, Gont LK and De Robertis EM: Xenopus chordin: A novel dorsalizing factor activated by organizer-specific homeobox genes. Cell 79: 779-790, 1994.

30. Lorenz TC: Polymerase chain reaction: Basic protocol plus troubleshooting and optimization strategies. J Vis Exp 63: e3998, 2012.

31. Jones CM and Smith JC: Wholemount in situ hybridization to Xenopus embryos. Methods Mol Biol 461: 697-702, 2008.

32. Yuan JS, Reed A, Chen F and Stewart CN Jr: Statistical analysis of real-time PCR data. BMC Bioinformatics 7: 85, 2006.

33. Rozen S and Skaletsky H: Primer3 on the WWW for general users and for biologist programmers. Methods Mol Biol 132: 365-386, 2000

34. Yoon J, Kim JH, Lee OJ, Yu SB, Kim JI, Kim SC, Park JB, Lee JY and Kim J: xCITED2 Induces Neural Genes in Animal Cap Explants of Xenopus Embryos. Exp Neurobiol 20: 123-129, 2011.
35. Session AM, Uno Y, Kwon T, Chapman JA, Toyoda A, Takahashi S, Fukui A, Hikosaka A, Suzuki A, Kondo M, et al: Genome evolution in the allotetraploid frog Xenopus laevis. Nature 538: 336-343, 2016

36. $\mathrm{Li} \mathrm{H}$ and Durbin R: Fast and accurate short read alignment with Burrows-Wheeler transform. Bioinformatics 25: 1754-1760, 2009.

37. Mi H, Huang X, Muruganujan A, Tang H, Mills C, Kang D and Thomas PD: PANTHER version 11: Expanded annotation data from Gene Ontology and Reactome pathways, and data analysis tool enhancements. Nucleic Acids Res 45 (D1): D183-D189, 2017.

38. Altschul SF, Gish W, Miller W, Myers EW and Lipman DJ: Basic local alignment search tool. J Mol Biol 215: 403-410, 1990.

39. Betancur P, Bronner-Fraser M and Sauka-Spengler T: Assembling neural crest regulatory circuits into a gene regulatory network. Annu Rev Cell Dev Biol 26: 581-603, 2010.

40. Sauka-Spengler T and Bronner-Fraser M: A gene regulatory network orchestrates neural crest formation. Nat Rev Mol Cell Biol 9: 557-568, 2008.

41. Ishii M, Merrill AE, Chan YS, Gitelman I, Rice DP, Sucov HM and Maxson RE Jr: Msx2 and Twist cooperatively control the development of the neural crest-derived skeletogenic mesenchyme of the murine skull vault. Development 130: 6131-6142, 2003

42. Huang C, Kratzer M-C, Wedlich D and Kashef J: E-cadherin is required for cranial neural crest migration in Xenopus laevis. Dev Biol 411: 159-171, 2016.

43. Bronner-Fraser M: Neural crest cell formation and migration in the developing embryo. FASEB J 8: 699-706, 1994.

44. Chen ZF and Behringer RR: twist is required in head mesenchyme for cranial neural tube morphogenesis. Genes Dev 9: 686-699, 1995.

45. Lin H, Zhu X, Chen G, Song L, Gao L, Khand AA, Chen Y, Lin $\mathrm{G}$ and Tao Q: KDM3A-mediated demethylation of histone H3 lysine 9 facilitates the chromatin binding of Neurog2 during neurogenesis. Development 144: 3674-3685, 2017.

46. Bellmeyer A, Krase J, Lindgren J and LaBonne C: The protooncogene c-myc is an essential regulator of neural crest formation in xenopus. Dev Cell 4: 827-839, 2003.

47. Monsoro-Burq A-H, Wang E and Harland R: Msx1 and Pax3 cooperate to mediate FGF8 and WNT signals during Xenopus neural crest induction. Dev Cell 8: 167-178, 2005

48. Ishimura A, Maeda R, Takeda M, Kikkawa M, Daar IO and Maéno M: Involvement of BMP-4/msx-1 and FGF pathways in neural induction in the Xenopus embryo. Dev Growth Differ 42: 307-316, 2000

49. Basch ML, Bronner-Fraser M and García-Castro MI: Specification of the neural crest occurs during gastrulation and requires Pax7. Nature 441: 218-222, 2006.

50. Mayor R, Young R and Vargas A: Development of neural crest in Xenopus. Curr Top Dev Biol 43: 85-113, 1999.

51. Dyson S and Gurdon JB: Activin signalling has a necessary function in Xenopus early development. Curr Biol 7: 81-84, 1997.

52. Aberger F, Weidinger G, Grunz H and Richter K: Anterior specification of embryonic ectoderm: The role of the Xenopus cement gland-specific gene XAG-2. Mech Dev 72: 115-130, 1998.

53. Tereshina MB, Zaraisky AG and Novoselov VV: Ras-dva, a member of novel family of small GTPases, is required for the anterior ectoderm patterning in the Xenopus laevis embryo. Development 133: 485-494, 2006.

54. Nakata K, Nagai T, Aruga J and Mikoshiba K: Xenopus Zic3, a primary regulator both in neural and neural crest development. Proc Natl Acad Sci USA 94: 11980-11985, 1997.

55. Doniach T and Musci TJ: Induction of anteroposterior neural pattern in Xenopus: Evidence for a quantitative mechanism. Mech Dev 53: 403-413, 1995.

56. Slack JM, Darlington BG, Gillespie LL, Godsave SF, Isaacs HV and Paterno GD: Mesoderm induction by fibroblast growth factor in early Xenopus development. Philos Trans R Soc Lond B Biol Sci 327: 75-84, 1990.

57. Tsukada Y, Fang J, Erdjument-Bromage H, Warren ME, Borchers $\mathrm{CH}$, Tempst $\mathrm{P}$ and Zhang Y: Histone demethylation by a family of JmjC domain-containing proteins. Nature 439: 811-816, 2006.

58. Shpargel KB, Starmer J, Yee D, Pohlers M and Magnuson T: KDM6 demethylase independent loss of histone H3 lysine 27 trimethylation during early embryonic development. PLoS Genet 10: e1004507, 2014

59. Kinoshita T, Haruta Y, Sakamoto $C$ and Imaoka S: Antagonistic role of XESR 1 and XESR5 in mesoderm formation in Xenopus laevis. Int J Dev Biol 55: 25-31, 2011. 